\title{
EMDR Therapy Treatment of Grief and Mourning in Times of COVID-19 (Coronavirus)
}

\author{
Roger M. Solomon \\ Arlington, Virginia, EMDR Institute \\ Barbara J. Hensley \\ Cincinnati, Ohio
}

\begin{abstract}
Death of a loved one is universally distressing. The stressful conditions of COVID-19 can compound the trauma of a loss. Consequently, the mourner has to deal with: (a) the loss of a loved one; (b) potential complications of grief and mourning caused by COVID-19 (e.g., sudden and unexpected death, a loved one's suffering, inability to be physically present to offer comfort or say good-bye, social distancing interfering with funeral and religious ceremonies); and (c) personal disruption caused by COVID-19 (e.g., disruption of employment and daily living routines, fears related to safety and uncertainty). Further, grief can be complicated by prior unresolved losses and trauma, including attachment-based trauma, which would also need to be identified and treated. This article presents a framework for treatment of grief and mourning with eye movement desensitization and reprocessing (EMDR) therapy. EMDR treatment, guided by the Adaptive Information Processing model, can be informed by other frameworks, including attachment theory and the Dual Process Model, which are described. A case example is presented to illustrate treatment of a client whose father died due to COVID-19.
\end{abstract}

Keywords: grief; mourning; EMDR; COVID-19

$\mathbf{T}$ his article focuses on the death of a loved one in the context of the COVID-19 pandemic. The pandemic has led to a plethora of losses for individuals, communities, states, countries, and the world at large. These layers of losses include death of loved ones, potential life-threatening illness, loss of physical contact with family and friends, loss of employment and financial security, loss of familiar routines and future plans, loss of safety and predictability, loss of control, and loss of personal freedoms. Systems we depended upon, such as our economic, education, and healthcare systems, continue to be in upheaval, and the impact continues to be felt worldwide. A study by Wang et al. (2020) focused on the psychological impact of the COVID-19 outbreak and found that $53.8 \%$ of respondents rated the psychological impact as moderate or severe; $16.5 \%$ of respondents reported moderate to severe depression symptoms; $28.8 \%$ of respondents reported moderate to severe anxiety symptoms; and $8.1 \%$ reported moderate to severe stress levels.

The loss of a loved one is universally distressing. Bereavement, even when uncomplicated, can have life-changing consequences (Osterweis, Solomon, \& Green, 1984; Solomon \& Rando, 2007, 2012, 2015). Further, the death of a loved one may trigger past traumas and losses and increase one's emotional vulnerabilities. The impact of the death of a loved one under COVID-19 is compounded due to restrictions, such as social distancing, inability to be present at the bedside of a loved one to comfort and to express one's love or make peace before the death, and the significant changes in how wakes, funerals, and religious ceremonies are practiced.

In this article we discuss the extra layers of difficulty associated with loss during COVID-19. First, there is the loss itself, a fact which in and of itself can be 
overwhelming. Secondly, current circumstances compound the trauma of the loss. Thirdly, there is the personal day-to-day disruption of everyday living caused by COVID-19. The interaction of these three variables can interfere with adaptation to the loss. All three layers need to be addressed.

\section{Grief and Mourning}

"For most people love is the most profound source of pleasure in our lives, while the loss of those whom we love is their most profound source of pain. Hence, love and loss are two sides of the same coin. We cannot have one without risking the other" (Colin Parkes, 2013, p. 1). When we lose someone we love, we may be losing a person we looked to for security, comfort, meaning, and balance in life. A loved one is both a part of our lives and external world and part of our identity and our internal world. Whether the relationship was positive or negative, secure or insecure, peaceful or conflictual, the deceased had a contributing role in defining who and what we are. In the immediate aftermath of a loss, especially when it is sudden and unexpected, it is difficult to fathom who we are or what life will be like without the loved one. With the loss of someone who is important, the world changes; things will never be the same.

Grief and mourning are different. Grief is the reaction a person has to loss. Mourning is the process of assimilation and accommodation to the loss and adapting to the world without the deceased. Neimeyer (2015) views grieving as a process of reconstructing a world that has been challenged by the loss. The death of a loved one creates a crisis of meaning in the life of the bereaved. Mourners need to process the narrative story of the death to "make sense" of what has happened, including the implications for ongoing life. Therefore, there is a reorientation of one's inner world, one's external world, and the relationship to the lost loved one (Rando, 1993). Consequently, there needs to be:

1. Personal adaptation to the loss. The relationship with a loved one can be a major part of one's identity and assumptive world, and has to be revised to the extent the mourner has been impacted by the death and its consequences.

2. Adaptation to the new world without the deceased. The external world and how one exists within it is different with the loss of a loved one.

3. A transformation of the relationship to the deceased. This relationship shifts from loving one in presence to loving one in absence (Attig, 2000).
Along with primary losses that result when a loved one dies, secondary losses affect us as well. These losses are a consequence of the primary loss which compound the hardship. For example, the death of a spouse often causes a secondary loss with decreased income for the surviving spouse and children. The other secondary losses in this situation might involve adapting to new geographical and social environments (e.g., being forced to move to a new, often lesser, house, leaving behind friends and losing the "old" neighborhood, and the children attending a new school).

\section{Loss of the Assumptive World}

The hallmark of trauma involves another major secondary loss - the loss of one's assumptive world. Death entails a drastic permanent change in the ongoing real relationship that is easily perceived yet cannot be immediately assimilated into one's assumptive world (Janoff-Bulman, 1989; Shear $\&$ Shair, 2005). Based on previous experience, the assumptive world, contains the mental schemata of everything one assumes to be true about the world, the self, and others. Violations of our basic assumptions (JanoffBulman, 1989) include: (a) It's a benign universe (i.e., "It's not supposed to happen, not to me"); (b) It's a meaningful world (i.e., "The world is supposed to be predictable, and fair"); and (c) The self is worthy (i.e., "bad things do not happen to good people, so what does this say about me?”).

The mourner's assumptive world can be significantly disrupted by the death of a loved one. As a result, Pearlman, Wortman, Feuer, Farber, and Rando (2014) report that mourners may:

- Have difficulty accepting the loss and making sense or grasping the meaning of the loss.

- Become preoccupied with causality, responsibility, and blame.

- Question faith.

- Become preoccupied with the deceased's suffering (e.g., what they were experiencing when they died).

- Experience guilt (especially parents who may have deep feelings of responsibility for their children).

COVID-19 has changed the world and how we live and, in the wake of its traumatic impact, may have violated one's assumptive world. Basic assumptions about safety, a meaningful world where there is control and predictability, and the self being worthy, may have been torn apart. Consequently, there is the trauma 
of the loss of a loved one, complicated with further trauma and loss from the impact of COVID-19.

\section{Traumatic Bereavement}

Traumatic bereavement occurs when the grief over a loved one is overpowered by the traumatic stress resulting from the circumstances of the death. It is characterized by enduring symptoms of both trauma and grief (Pearlman et al., 2014). The traumatic nature of an unexpected death is compounded by COVID-19 is compounded by social distancing and one's inability to be physically present at the time. Consequently, the mourner is forced to deal with the trauma of the loss and the trauma of the circumstances that surround it.

Below are some risk factors that affect the response to the death and influence the level of grief and mourning (Pearlman et al., 2014; Worden, 2018;). The more of these factors that are involved, the more difficult it can be to comprehend what happened and to begin dealing with it. However, it is important to realize that there are individual differences on the impact of these factors according to one's perception of the event, prior history of trauma and loss, and coping ability.

1. Suddenness and lack of anticipation. Contracting COVID-19 and dying as a result can be sudden and unexpected. The expectation and hope for recovery can make the death more devastating.

2. Unnaturalness of the death. There are many atypical occurrences associated with COVID-19. Visitation with COVID-19 patients is strictly prohibited. Friends and family are unable to be present to hold the loved one's hand or say a last "goodbye" at the time of passing. Funerals must be watched from afar or delayed for weeks or months, depriving friends and family of the traditional rituals and moments of togetherness so important and comforting to the grieving process.

3. Physical or emotional suffering. It is distressing to watch the loved one suffer when there is nothing one can do. This sense of helplessness and powerlessness is magnified by an inability to provide faceto-face comfort.

4. Preventable deaths. There is a different reaction to deaths seen as unavoidable (e.g., old age) and those regarded as preventable (e.g., due to negligence or carelessness). With COVID-19, some may view the death as preventable for a variety of reasons: (a) the government's delayed response to the severity of the COVID-19 threat; (b) lack of sufficient personal protective equipment (PPE) to healthcare facilities and workers; (c) nonexistent or insufficient preventive guidelines or protection at workplaces; or (d) disregard by the deceased of recommended safety precautions (e.g., wearing a mask, social distancing) or e) mourners may wonder if they could have done something to prevent the death (e.g., "If only I did something different, they would have lived"). Mourners often struggle with the senselessness of a death and may experience strong feelings of anger (e.g., "It was preventable and should not have happened.").

5. Randomness. The randomness of an event may best be characterized by "being in the wrong place at the wrong time." COVID-19 is an invisible enemy and, therefore, uncontrollable. Even with lockdown conditions and restricted movement, one can still contract it at the grocery store, gas station, or from a family member. The presumed randomness of catching the virus and dying can complicate the mourning.

6. Multiple deaths. Multiple deaths may occur within one household or among close friends. This can create a "bereavement overload," (Rando, 1993, 2015) complicating the mourning process.

7. Threat to one's own life/Confrontation with the death of others. If the mourner's life was threatened by the same circumstances that caused a loved one's death, several difficult issues can compound and complicate the grief process. In these cases the mourner must deal with: (a) personal feelings of vulnerability and powerlessness over the loss of a loved one; (b) an increased sense of mortality if the mourner also contracts the virus; and (c) potential guilt over one's inability to prevent the death and/or survivor guilt (e.g., "I feel guilty for surviving; it should have been me").

8. Untimeliness. All losses are terrible, but research (Pearlman et al., 2014) has shown that the death of a child can have more impact than other losses. When a child or a young adult dies, intense feelings of anger, injustice, and sadness are often felt due to the loss of a life of unrealized potential. Even older adults with preexisting conditions can be robbed of extra life by COVID-19, making the death untimely.

9. Social support. Social support is an extremely important resource when dealing with distressing life experiences. In these days and nights of quarantine and social distancing, mourners cannot have direct, face-to-face rituals, such as religious ceremonies, funerals, and visits from friends and family. Lack of social support can compound the trauma and grief of the loss. 
There are other notable risk factors that complicate grief that may not be as applicable to COVID-19. These factors include:

1. Violence. Violent deaths (e.g., homicide, suicide) typically evoke shock or horror, which may overwhelm one's sense of control and violate one's assumptive world. Although not the direct or deliberate result of a violent action perpetrated by another, there may be an element of violence associated with COVID-19 due to the swiftness of the illness, the pain and suffering it causes, and the last virtual images of the loved one.

2. Intentional death. Death caused by a perpetrator who intentionally killed the loved one goes beyond the emotional impact of preventable deaths. Intentional acts of violence (e.g., murder, terrorism, suicide, domestic violence) can be more potentially traumatizing because they are the result of deliberate and malicious intent and action. Acts of violence significantly disrupt the mourner's assumptive world, undermining faith and trust in people, creating the realization of evil, and evoking rage, powerlessness, and vulnerability.

3. Ambiguous deaths. Tragedies, such as oceanic airplane crashes, mass casualties, explosions, or fires; can result in no tangible evidence of death (i.e., no physical remains). Lack of physical confirmation of a loved one's death can impede the mourning process and prevent a sense of closure, leaving a lingering question in the air (i.e., "Did my loved one really die?"). When the cause of death remains unknown or ambiguous, the mourner may become preoccupied with understanding how the loved one died. The nature of COVID-19 illustrates this potential ambiguity.

4. Stigmatized deaths. Death by suicide or by AIDS is often seen as stigmatizing. This stigmatization can result in feelings of shame and interferes with one seeking and receiving social support.

\section{EMDR Therapy}

Eye movement desensitization and reprocessing (EMDR) therapy is a therapeutic approach that can be effective in the treatment of grief and facilitate the mourning process (Shapiro, 1997; Solomon $\&$ Rando, 2007, 2012, 2015). In a random controlled study (Meysner, Cotter, \& Lee, 2016) EMDR therapy was compared with integrated cognitive behavior therapy (CBT). Both interventions were found to be equally effective. Cotter, Meysner, and Lee (2017), presenting interview data from the same study, reported both groups showed a heightened "mental" relationship with the deceased, positive shift in emotions, and increased insight, activity levels, and self-confidence. Hornsveld et al. (2010), acknowledging previous studies showing eye movements reduced the emotionality of negative memories, investigated the effect of eye movements (as used in EMDR therapy) on loss-related negative memories. It was found that recall of the negative memory plus eye movement was more effective than "no stimulation" or listening to music. Sprang (2001) demonstrated that EMDR therapy is effective with complicated mourning. This study, comparing EMDR and Guided Mourning in the treatment of complicated mourning, found that EMDR resulted in a significantly greater reduction on four of the five psychosocial measures of distress. Further, positive memories of the loved one emerged during EMDR treatment. This did not occur with Guided Mourning.

Case studies and discussion on the utilization of EMDR with grief and mourning have been presented by Murray (2012) and Yasar, Abamor, Usta, Tay-can, and Eroglu (2017). Solomon and Rando (2007, 2012) discussed how EMDR therapy can be utilized in the treatment of grief and mourning guided by the framework of Rando's " $R$ " processes (Rando, 1993). The utilization of EMDR with grief and mourning has also been discussed by Solomon and Shapiro (1997), Kimiko (2010), and Solomon (2018).

\section{Grief in Terms of the Adaptive Information Processing Model}

The Adaptive Information Processing model (AIP) (Shapiro, 1995, 2001, 2018) views present problems as the result of distressing memories becoming maladaptively stored (i.e., stored in state-specific, excitatory form in its own neural network) in the brain and unable to process. The maladaptively stored memory can continue to be triggered by a variety of internal and external stimuli, resulting in inappropriate emotional, cognitive, and behavioral reactions as well as overt symptoms (e.g., high anxiety, nightmares, intrusive thoughts). EMDR memory processing involves accessing the memory and stimulating the information processing system through bilateral stimulation (e.g., eye movement, taps, tones). This enables adaptive information to link into the memory network holding the maladaptively stored memory and facilitating integration of the memory into the wider memory network. The trauma of a major loss can result in many moments, situations, and memories, (e.g., getting the news, hospital images, funeral scenes) becoming maladaptively stored. When the loss 
is triggered (e.g., thinking about or being reminded of the deceased), anguish, pain, and difficulties in adaptation result. The loss can be so distressing that other memory networks with positive memories of the loved one cannot be accessed, experienced, and felt. The mourner may have a sense of "I can't connect" to the deceased because the emotional pain is so overwhelming.

\section{EMDR Therapy and Grief ("I Can't Connect" to "I Can Connect")}

EMDR processing facilitates the expression, experiencing, and discharge of the pain that comes with loss. This is necessary for the eventual linking in of positive, adaptive information. Clinicians who use EMDR with mourners consistently observe the emergence of positive memories of the deceased with its associated affect (Solomon \& Rando, 2007, 2012, 2015). Sprang (2001), cited above, also observed the emergence of positive memories. The heartfelt, positive memories that arise provide a sense of connection to the loved one. According to Continuing Bonds theory (Marwit $\&$ Klass, 1996), healthy adaptation to loss occurs when mourners are able to internalize a representation of the deceased into an inner working model enabling psychological proximity to substitute for the previous physical proximity. EMDR therapy seems to facilitate the formation of an adaptive inner representation with movement from "I can't connect," with its consequent deep pain, to "I can connect," with the emergence of heartfelt memories providing a sense of connection to the loved one.

If there was a conflictual history with the deceased, the inner representation may not be positive. Unresolved conflicts and trauma that involved the deceased must still be processed and resolved. EMDR therapy can be used to process the negative memories that contributed to a toxic relationship and treat the unresolved relational trauma and attachment memories that complicate mourning and continue to create present problems.

\section{EMDR Therapy Is a Natural Healing Process}

EMDR therapy proceeds in a way that is natural for the mourner. It does not take away what is appropriate to the situation, what one needs, or what is true (Shapiro, 2018; Solomon, 2018; Solomon \& Shapiro, 1997). As Shapiro (2018) stated, "EMDR does not eliminate or even dilute healthy, appropriate emotions, including grief. Rather, it can allow clients to mourn with a greater sense of inner peace” (p. 232). Consequently, EMDR can be utilized to treat the painful distress that accompanies uncomplicated grief, including what are considered to be normal reactions. For example, it is normal to be upset by intrusive imagery (e.g., hospital scenes). EMDR therapy can target these distressing moments and process the pain and facilitate integration in a way that is helpful and natural. EMDR therapy processes the obstacles that can complicate the grief, enabling progression through the mourning process (Solomon \& Rando, 2007, 2012, 2015; Solomon, 2018).

\section{Complicated Grief and Mourning}

Experiencing the mourning process depends on the mourner's ability to endure the emotional pain of recognizing that the loss is irrevocable (e.g., the loved one is gone and never coming back). This can be overwhelming for some. The trauma of the death and the complications due to COVID-19 can certainly complicate the grief and mourning process. Research has shown by 6 months most bereaved individuals get through the initial sense of disbelief that comes with being overwhelmed and are able to accept the loss as a reality, eventually re-establish a new equilibrium after loss without developing any prolonged impairment and proceed with daily life (Jordan \& Litz, 2014). However, between $10 \%$ to $20 \%$ of bereaved people suffer from severe and disabling grief for a prolonged period (Prigerson, Vanderwerker, \& Maciejewski, 2008).

Complicated grief reactions involve the mourner's attempt to: (a) avoid, deny, or repress aspects of the loss, its pain, and the full realization of the implications of the death; and/or (b) hold on to and avoid letting go of the deceased loved one (Rando, 1993). Complicated grief is characterized by an intense yearning and longing for the loved one who has died (Prigerson et al., 2008) leaving the mourner feeling empty, having little hope for the future, and being preoccupied by sorrow and regrets concerning the loss. Along with intrusive and distressing thoughts regarding the loved one's absence, ruminating on the death makes it difficult for one to move beyond an acute state of mourning.

\section{Other Frameworks}

Several theoretical frameworks enable us to understand grief and mourning, how complications develop and guide EMDR therapy treatment planning and 
intervention. Neimeyer's (2015) social constructionist perspective was touched upon in the introduction, and Continuing Bonds (Marwit \& Klass, 1996) was mentioned in the section on EMDR and grief and mourning. Both are useful frameworks for understanding the process of grief and mourning. Rando's $\mathrm{R}$ processes (Rando, 1993) are helpful in describing the adaptation to loss, particularly when there are traumatic circumstances. The $\mathrm{R}$ processes provide a way to understand the progression of mourning and where to intervene if the mourning process becomes "stuck." Previous articles (Solomon \& Rando, 2007, 2012, 2015) have discussed the application of EMDR therapy to facilitate movement through the $\mathrm{R}$ processes. In this article, other models will be presented that can enhance understanding of grief and mourning and guide EMDR therapy. These include Attachment Theory and Dual Process Model (DPM).

\section{Attachment Theory}

Attachment orientation plays a significant role in how people adapt to loss and explains the individual differences in grief and mourning (Kosminsky $\&$ Jordan, 2016). The loss of a loved one can activate the responses and feelings that were experienced in childhood when there was separation from an attachment figure. Attachment styles result from early childhood interactions with the caregiver and can be conceptualized (in terms of the AIP model) as memory networks that are organized around child-caregiver interactions and provide a foundation of emotional information about self and other, thereby influencing relationships. Consequently, attachment theory can guide the EMDR clinician in the identification and treatment of the maladaptively stored memories that can complicate the grief. This model has the most implications for EMDR therapy, complicated mourning, and trauma with its emphasis on the role of early experiences.

Attachment styles form early in life and result from child-caregiver bonding. Infants come into this world hardwired to attach to caregivers (e.g., the parents) for both physical protection and a psychological sense of safety (Bowlby, 1960, 2005, 2008). When the caretaker provides "good enough" soothing and comfort and is able to meet the child's needs, the attachment system deactivates and resets (Kosminsky \& Jordan, 2016). A secure attachment style develops. If the child's distress signals, such as crying, do not bring the caretaker into proximity or the caregiver behaves in an angry, impatient, or rejecting manner in response to the child's disturbance, then secondary strategies come into play to reduce the distress (Bowlby, 1960, 2005, 2008; Mikulincer \& Shaver, 2016). These secondary strategies involve either hyperactivating or deactivating of the attachment system.

These secondary strategies of hyperactivation or deactivation of the attachment system become the child's optimal means for restoring or maintaining proximity to the caregiver (Kosminsky \& Jordan, 2016; Mikulincer \& Shaver, 2016). If the caretaker is perceived as comforting but is not always available or is inconsistent in providing comfort, then hyperactivating strategies have the best chance to keep the caregiver close. Hyperactivating strategies are attempts to keep the caregiver's proximity through clinging, crying, or protesting and showing distress when imminent separation is perceived. This is the precursor to an anxious attachment style. On the other hand, if the caregiver is perceived as consistently not being available or unable to meet needs (e.g., neglectful, critical, annoyed), then deactivating strategies (i.e., downregulating the attachment system) are the best way to avoid distress and discomfort (Mikulincer \& Shaver, 2016). Deactivating strategies involve a shutting down of awareness of discomfort and signaling behavior aimed at bringing the caregiver into proximity. The child may not only stop expressing discomfort but may stop feeling it. Deactivating strategies are the precursor to an avoidant attachment style.

Attachment and Grief. Loss of a loved one activates the attachment system and evokes many of the same reactions that accompanied separation from an attachment figure in childhood (Kosminsky \& Jordan, 2016). The loss is irreversible. This makes primary strategies for seeking comfort and safety from the deceased no longer relevant. Secondary strategies involving activation and deactivation of the attachment system must come into play. Consequently, attachment style is a major determinant of how one grieves and accounts for variations in the grief response (Kosminsky \& Jordan, 2016; Neimeyer, 2015; Parkes \& Prigerson, 2010; Wayment \& Vierthaler, 2002). Securely attached people can be greatly impacted and saddened by the death of a loved one but are likely to have an easier time adapting in comparison to those with insecure attachment styles (Mikulincer \& Shaver, 2016). The adage, "time heals all wounds," applies. However, individuals with insecure attachment styles in comparison to secure attachment may have more intense and persistent grief reactions. Research has demonstrated that mourners with anxious attachment styles, in comparison to secure attachment, are more likely to be hyperaroused and ruminate about the loved 
one, show clinging behavior, experience loneliness, and have overwhelming negative affect. (Kosminsky $\&$ Jordan, 2016; Mikulincer \& Shaver, 2016). Mourners with an avoidant attachment style tend to be numb and shut down. They appear to be doing well but can actually be experiencing internal distress (Parkes, 2013). When triggered, they may feel that they are being flooded with unwelcome, distressing emotion (Meier, Carr, Currier, \& Neimeyer, 2013).

Implications for EMDR Therapy. Complicated grief and mourning occur when the mourner attempts to: (a) deny or avoid the full realization and implications of the death; and / or (b) hold on to and avoid letting go of the lost loved one. The mourner's pain and difficulty accepting the loss can be likened to the infant/child who is preoccupied with reestablishing proximity to a caregiver (Kosminsky \& Jordan, 2016).

Insecure attachment styles (e.g., anxious, avoidant, disorganized) are not only determined by major distressing experiences (e.g., neglect, abuse), but also the ubiquitous, "seeming small" but impactful moments (e.g., "Mommy did not look at me when I was upset."). Therefore, treatment of complicated mourning involves treating both the trauma of the loss (e.g., focusing on the moment of shock or realization of the loss) and maladaptively stored child-caregiver interactions that can underlie current difficulties. Present triggers also need to be identified and processed and a future template for each trigger installed.

Attachment style also plays a significant role in how one responds to traumatic events and stressful circumstances, including COVID-19. The response is significantly influenced by past trauma, losses, and attachment-based negative memories (Afifi, Mota, Dasiewicz, MacMillan, \& Sareen, 2012; Felitti et al., 1998; Mol et al., 2005). The loss of face-to-face contact, social distancing, disruption of usual schedules, and financial hardship can trigger past attachment trauma, negatively impacting current functioning and compound the impact of the death of a loved one.

\section{DPM}

The DPM (Stroebe \& Schut, 2008) views healthy adaptation to loss as involving an oscillation (i.e., a shifting back and forth) between Loss Orientation (LO) - dealing with the pain of the loss, and Restoration Orientation (RO) - avoiding the pain and dealing with the practical and psychological issues pertaining to a future life without the loved one. The loss of a loved one activates the attachment system. With the death, primary strategies of seeking safety and comfort from the deceased are no longer viable. Secondary strategies come into play and involve activation (LO) and deactivation (RO) of the attachment system. LO strategies involving activation of the attachment system include searching, yearning, remembering, experiencing the presence of the loved one, and imaginal conversations. RO strategies involve a deactivation of the attachment system, with the mourner avoiding and turning away from the grief in order to cope with daily life tasks. The coping process involves an oscillation where intervals of turning away from the grief to deal with daily living is as necessary in the mourning process as moving toward and through the grief (Kosminsky \& Jordan, 2016; Strobe \& Schut, 1999, 2010).

Implications for EMDR Therapy. The DPM illustrates the importance of taking a broad view of intervention, focusing both on dealing with the emotional impact of the loss (LO) and how the mourner is functioning in daily life (RO). Some clients, overly focused in LO, may initially need interventions that help them oscillate toward RO. Focusing on affect management and regulation strategies, building and enhancing resources (e.g., Resource Development and Installation [Korn and Leeds, 2002]), and providing needed strategies and skills for coping with daily living tasks are examples. Clients that are overly focused in RO may need initial support to gradually approach the reality of the death with its accompanying thoughts and emotions. Taking the time to build a trusting relationship, perhaps using photos or videos to help the mourner get in touch with and express emotions, and building capacity and skills to tolerate emotion can bring about balance between LO and RO.

The stressful circumstances of COVID-19 may impact client functioning and ability to cope and interfere with the oscillation and balance between LO and RO. The balance and oscillation between one's ability to deal with the emotional impact of the loss (LO) and the ability to deal with day-to-day realities (RO) need to be assessed. With more than usual stressful circumstances, more emphasis may need to be put on RO in order to help the client deal with day-to-day life, problem solving, coming up with new life routines, and affect regulation strategies.

\section{Case Conceptualization for Loss in Times of COVID-19}

According to the AIP model, present-day problems are the result of memories maladaptively stored. EMDR 
therapy aids a client in processing the negative memories underlying present difficulties and the present triggers that evoke the distress in addition to installing a positive future template for each present trigger. The death of a loved one in the times of COVID-19 can create three levels of problems:

1. The loss of a loved one.

2. Traumatic or complicating circumstances due to COVID-19 (e.g., risk factors, such as a sudden, unexpected, untimely death involving suffering) and/or previous unresolved losses or traumas, including attachment-based trauma).

3. The impact of COVID-19 on personal functioning.

Exacerbated by risk factors caused by COVID-19, the loss of a loved one can interfere with one's ability to cope with current stressful circumstances, and these current stressful life circumstances can interfere with going through the mourning process. Consequently, treatment must deal with the loss, traumatic components of the loss, and personal functioning in the present stressful circumstances.

\section{EMDR's Eight Phases of Treatment}

EMDR is an eight-phase, three-pronged protocol (i.e., past, present, future) for treating distressing memories. The application of EMDR therapy to excessive grief has been described by Shapiro (2018) and Luber and Shapiro (2009) and is implemented in the case study described below.

\section{Phase 1: History}

On first contact, EMDR treatment begins with history taking, creating an opportunity to build therapeutic rapport and trust. History taking also involves therapeutic rapport building, understanding and empathic response, and gathering information according to the client's pace and readiness.

1. Personal impact of the loss (e.g., nature of the attachment with the deceased, strength of the relationship, security, conflicts, unresolved issues).

2. History taking should include an assessment of the client's current living situation and how COVID19 has impacted their life. This information can be gathered before, during, and after the following points are discussed. These topics are suggested for discussion and not in any ordered sequence.

3. Cognitive, emotional, physiological, and behavioral reactions to the death.

4. Risk factors for complicated grief.
5. Changes in the client's life since the death of the loved one.

6. Client's ability to cope with current circumstances.

7. Client's ability to regulate affect and maintain dual awareness.

8. In terms of the DPM, overall assessment of client functioning. This would evaluate the balance (i.e., oscillation) between LO (i.e., the client dealing with the emotional impact of the loss) and RO (i.e., the client's ability to deal with everyday-living issues).

9. Present triggers and reactions to reminders.

10. Trauma and loss history of relationships with close attachment figures.

It is imperative the clinician be appropriately trained to be able to assess the client for complex trauma and dissociation. Especially with increased usage of teletherapy during the COVID-19 pandemic, precautions and extra steps need to be put into place to ensure the client with complex trauma and dissociative symptoms is sufficiently stabilized for treatment.

Case Example-Jerry. Jerry is a 46-year-old man, and after COVID-19 restrictions were imposed, isolated himself at home with his wife and two young adult children. His father contracted COVID-19 illness, was hospitalized 1 week later, and died 1 week after that. As Jerry and his family had not seen the father in over 3 weeks, they felt confident that they were not at risk from the father's illness. The family sought treatment from a family therapist 3 weeks after his father died, and, as a result, Jerry was referred for individual treatment.

Jerry knew that his father could die, but his death was still a shock because of his hope for his father's recovery. It was distressing to be at such a distance, watching his father's rapid decline in health and obvious discomfort on a computer screen, talking to him and saying good-bye online, unable to adequately communicate his emotions and love. The funeral service was held online with only the family members and the chaplain present to view his father's closed casket. Obviously, no touching or hugging or funeral reception was allowed. At the graveyard, with no other people in attendance, the family watched the burial service from their car. An online memorial service and online chats with friends and family were helpful, even heartwarming, but also troubling to Jerry and his family because of how distant and impersonal it felt with the lack of physical proximity.

Jerry presented with trauma symptoms of intrusive images of his father in the hospital, nightmares (e.g., trying to talk to his father but in his dream he 
was unable to speak), difficulty concentrating on his work, anxiety, and feelings of powerlessness because he could not do anything or be physically present for his father. The computer from which he worked was the same computer he used to talk with his father and would trigger images of his father's suffering. Further, since his father's death he felt insecure while working, He was experiencing self-doubt about his capabilities. Spending more time alone and not engaging in his usual level of conversation, he also was becoming more withdrawn from his family.

Jerry described an ambivalent relationship with his father. His father was loving and provided for the needs of the family when Jerry was growing up but also had a quick temper and frequently yelled at the children. Jerry reported he always had a negative belief of "I'm not good enough," which he understood was linked to negative memories involving his father. Specific memories of his father yelling at him and his brother were identified. Further, the memory of his grandfather's death was also triggered with the father's illness. Jerry had been close to his grandfather, who lived with the family, and died when Jerry was 6 . Jerry was alone with his grandfather when he passed and still had upsetting memories of this incident. Jerry and his father had established a good relationship after Jerry got married.

Jerry's Case Conceptualization. Jerry's oscillation between LO and RO was off balance. His distress at the loss of his father was compounded by COVID-19 risk factors and the triggering of previous losses and attachment-based memories. Jerry was more focused on LO with his suffering from the loss of his father. This interfered with his ability to concentrate on his work and engage with his family (RO). Risk factors in Jerry's case included: (a) sudden and unexpected death; (b) unnaturalness of the death; (c) the father's suffering; (d) randomness; and (e) an untimely death.

Jerry had an anxious attachment with his father. His insecurities and negative self-belief ("I'm not good enough") stemmed from childhood and heightened after the death of his father, interfering with work and his emotional availability to his family.

Jerry's treatment plan was as follows:

- Target memories related to the immediate death:

1. The last time he saw his father alive in the hospital on the respirator (i.e., watching from his computer).

2. A moment where he felt helpless when talking to his father over the computer.

3. Images of his father suffering.
4. The funeral, not being able to hug immediate friends and family members who attended, and images of watching the burial from his car.

- Target memories stemming from previous losses and (attachment) trauma:

1. The death of the grandfather.

2. Memories of his father yelling at him and his brother.

- Target present triggers:

1. Being at his computer while working and having images of his father's suffering coming up.

2. Moments where he wanted to be more talkative and involved with his family at home but found it difficult.

3. Distressing feelings when thinking about his father because of the negative images related to his death.

- Future Templates:

1. Being able to work comfortably on the computer.

2. Being able to engage comfortably with his family.

3. Being able to think of his father with a sense of peace and love.

\section{Phase 2: Preparation}

The Preparation Phase ensures the client has sufficient stabilization and integrative capacity to begin EMDR memory processing. The client is also educated about EMDR therapy and procedures. Some clients may need specific sessions devoted to coping with the stressful impact of COVID-19 before processing memories related to loss. The client needs to have sufficient integrative capacity and affect-regulation strategies for coping with the often-intense emotions both during the session, after the session, and in-between sessions. This is especially important in this era of virtual sessions.

The readiness criteria for EMDR memory processing include, to a good enough level, the ability to:

1. Access the experience and stay present (i.e., maintain dual awareness between past experience and present situation).

2. Observe the experience and reflect on it rather than be completely absorbed by it.

3. Tolerate distress for a short period without becoming overwhelmed (i.e., hyperarousal) or shutting down (i.e., hypoarousal). 
4. Shift emotional states (e.g., from distress to calm) and access positive experiences and resource states.

5. Practice self-soothing, containment, and adaptive coping strategies and skills in between sessions as needed.

6. Create a stable (and safe) social environment.

7. Experience sufficient trust and safety in the therapeutic relationship.

Case Example-Jerry (Continued). Jerry was given four online preparation sessions within a 2-week period involving history taking and affect management. The Safe/Calm Place and breathing exercises were helpful with affect management. Resource Development and Installation (Korn \& Leeds, 2002) helped Jerry deal with working from his computer. Implementation of a more structured daily routine was also beneficial, focusing on his work schedule with breaks, daily exercise through an online program that he and his wife could do together, and meal and leisure time with the family. This assisted Jerry in maintaining a more balanced oscillation between $\mathrm{LO}$ and $\mathrm{RO}$ and meet criteria for memory processing.

Phase 3-6: Assessment, Desensitization, Installation, and Body Scan

Phase 3 is a structured process designed to activate the memory network. In relation to the memory, the following information is identified: (a) the worst image; (b) negative cognition (i.e., irrational belief about the self); (c) positive cognition (i.e., the preferred belief about the self); and (d) emotions and sensations. Subjective measures are assessed which include the Validity of Cognition (i.e., how true the positive cognition feels when thinking of the memory on a 1-7 scale, with one totally false and 7 totally true) and the Subjective Units of Disturbance (i.e., $0-10$ how disturbing it is with 0 being neutral or calm and 10 being the worst imaginable).

In Phases 4 through 6, the memory is desensitized, cognitive reprocessing occurs, and the somatic distress is relieved. Sometimes processing gets stuck because past traumas or losses, including seemingly small attachment-related memories, are being triggered. Floatback or affect scans (Shapiro, 2018) can be used to identify memories that underlie blocks in processing. For example, a client got stuck when processing the memory of making the decision to take his mother off life support. Despite repeated sets of bilateral stimulation, the stuck point (i.e., "Now I will never have the love I have always wanted from my mother.") would not resolve. A floatback identified past memories related to his statement. After processing these memories, the original target concerning the mother's death could be fully processed.

Strengthening Positive Memories. The result of processing the loss of a loved one is the transformation of the relationship from loving in absence to loving in presence. This is seen in the emergence of positive, heartfelt memories that form an adaptive inner representation. Should this be the case, it may be beneficial after the body scan to enhance the adaptive inner representation by having the client think of the loved one and doing several sets of bilateral stimulation (Solomon, in press). The sets should be shorter and slower than usual, which is the proper procedure for installing the Safe/Calm Place or other resourcing techniques.

Case Example-Jerry (Continued). The treatment plan outlined above was followed. The initial target was Jerry seeing his father for the last time on the respirator and not being able to be present to offer comfort. This treatment session was tearful, with Jerry remembering his final conversation with his father before he went on the respirator. With further processing, he felt at a deep level that his father knew he was not alone and that Jerry loved him. Positive and heartfelt memories of his father alive also came to mind. After the body scan, when asked to think of his father, positive memories came up and were enhanced with brief sets of bilateral stimulation.

During processing of a painful memory of his father's suffering, the memory of his grandfather's death emerged. Jerry was alone in the room with him when he died. The associative result was a positive shift from a frightful image of his dead grandfather to a peaceful one to being able to think of his father at peace as well. With continued processing, positive memories of his father emerged, illustrating how EMDR processing facilitates an adaptive inner representation of the deceased. In subsequent sessions, other surfacing memories (e.g., childhood memories of his father yelling at his brother and himself) were also processed and resolved.

As Jerry continued to be triggered when he worked on his computer, this frequent daily occurrence was processed using the standard EMDR therapy protocol. Once his anxiety had been desensitized, a future template was installed. Jerry was also unable to be alone and to engage in conversation with his family without being triggered. This trigger was processed and resolved, and a future template where he visualized 
being able to comfortably interact with his family was installed.

\section{Phase 7: Closure}

Particularly with clients suffering from complex trauma and dissociation, closure is particularly important in this era of COVID-19. Clients, especially those who live alone, or are caring for others, need to be grounded and stable before leaving a session. A review of the stabilization and coping strategies taught in the Preparation Phase, as well as contingencies for dealing with issues that may have arisen during the session, should be addressed.

Case Example-Jerry(Continued). At the end of the first processing session, Jerry was tearful. When processing the memory of saying goodbye to his father was resolved, he felt a greater sense of peace and connection. After a review of affect-regulation and coping strategies and a reminder of his safe place, the session ended. Each session appeared to less emotional for Jerry and to leave him grounded and stable.

\section{Phase 8: Reevaluation}

At the beginning of each session, the clinician and client review what has taken place since the last session, and the results of the previous session are evaluated. Particular attention should be paid to how the client handled the session during the week. Attention should be given to what came up during the week and how it impacted their ability to cope with present circumstances. During this phase, the target is selected for that session and processing continues with Phases 3 through 6 .

Case Example-Jerry (Continued). Each session began with a review of what had happened since the last session and inquiry about the results of the previous processing session. Jerry consistently reported feeling better. He was able to concentrate and be more involved with his family. The oscillation between LO and $\mathrm{RO}$ progressively became more balanced.

\section{Termination}

After completing the processing of memories related to his father's death and past childhood memories, present triggers, and installing future templates, Jerry had a balanced oscillation between LO and RO. Though he was sad and missed his father, he could think of his father with a sense of peace and reflect on meaningful, positive memories of him. Jerry was more engaged with his family, adapted to the new schedule, and was able to be focused while working. Treatment terminated with an agreement to check back if he felt he was getting out of balance.

\section{Discussion}

The three layers consisting of Jerry's loss, the compounding factors caused by COVID-19, and the distress emanating from life changes due to COVID19, were treated with EMDR therapy. Focusing on enhancing his RO, the Preparation Phase involved helping Jerry cope with day-to-day life resulting from COVID-19. This included affect-regulation strategies and implementing a more structured daily routine. The trauma of his loss involved targeting the worst moments and present triggers and installing future templates. It was also important to identify and process past unresolved losses (e.g., the grandfather's death) and negative memories involving his father, further enabling adaptation to his loss and present circumstances. With processing of distressing memories, positive heartfelt memories emerged, illustrating the formation of an adaptive inner representation. This gave Jerry a positive sense of connection with his father, helping in the transformation of the relationship from loving in presence to loving in absence.

\section{Conclusion}

The loss of a loved one is painful, and grief and mourning can be complicated by the distressing circumstances of the COVID-19. Further, unresolved losses and trauma, including attachment-based memories, can contribute to the client's symptoms and reactions to present loss. EMDR therapy, guided by the AIP model and other theoretical frameworks pertinent to grief and mourning, can be utilized to treat the trauma of the loss, current distressing circumstances, and the underlying memories contributing to the current clinical picture. EMDR therapy facilitates integration of traumatic memories and enhances present functioning through processing of present triggers and the laying down of future templates. In this context, EMDR can also be understood as a paradigm of resilience, enabling positive adaptation to difficult circumstances.

\section{REFERENCES}

Afifi, T. O., Mota, N. P., Dasiewicz, P., MacMillan, H. L., \& Sareen, J. (2012). Physical punishment and mental disorders: Results from a nationally representative 
US sample. Pediatrics, 130, 1-9. doi:10.1542/peds. 2011-2947

Attig, T. (2000). Anticipatory mourning and the transition to loving in absence. In T. A. Rando (Ed.), Clinical dimensions of anticipatory mourning. Research Press.

Bowlby, J. (1960). Grief and mourning in infancy and early childhood. Psychoanalytic Study of the Child, 15(1), 9-52. doi:10.1080/00797308.1960.11822566

Bowlby, J. (1982). Attachment and loss: Retrospect and prospect. American Journal of Orthopsychiatry, 52(4), 664-678. https://doi.org/10.1111/j.1939-0025.1982. tb01456.x.

Bowlby, J. (2005). A secure base: Clinical applications of attachment theory. Taylor and Francis.

Bowlby, J. (2008). A secure base: Parent-child attachment and healthy human development. Basic Books.

Cotter, P., Meysner, L., \& Lee, C. W. (2017). Participant experiences of eye movement desensitisation and reprocessing vs. cognitive behavioural therapy for grief: Similarities and differences. European Journal of Psychotraumatology, 8, 1-9. doi:10.1080/ 20008198.2017.1375838

Fairbairn, W .D. (1952). An object-relations theory of the personality. Basic Books.

Felitti, V. J., Anda, R. F., Nordenberg, D., Williamson, D. F., Spitz, A. M., Edwards, V., \& ... Marks, J. S. (1998). Relationship of childhood abuse and household dysfunction to many of the leading causes of death in adults: The adverse childhood experiences (ACE) study. American Journal of Preventive Medicine, 14, 245258. doi:10.1016/S0749-3797(98)00017-8

Hornsveld, H. K., Landwehr, F., Stein, W., Stomp, M., Smeets, M., \& Van den Hout, M. (2010). Emotionality of loss-related memories is reduced after recall plus eye movements but not after recall plus music or recall only. Journal of EMDR Practice and Research, 4, 107-112. doi:10.1891/1933-3196.4.3.106

Janoff-Bulman, R. (1989). Assumptive worlds and the stress of traumatic events. Social Cognition, 7, 113-136. doi:10.1521/soco.1989.7.2.113

Jordan, A. H., \& Litz, B. T. (2014). Prolonged grief disorder: Diagnostic, assessment, and treatment considerations. Professional Psychology: Research and Practice, 45(3), 180187. doi: 10.1037 /a0036836

Kimiko, S. (2010). The effect of PTSD treatments after stillbirth: Eye Movement Desensitization and Reprocessing (EMDR) combined with hypnotherapy. Journal of Psychosomatic Obstetrics and Gynecology, 31, 98.

Korn, D., \& Leeds, A. (2002). Preliminary evidence of efficacy for EMDR resource development and installation in the stabilization phase of treatment of complex posttraumatic stress disorder. Journal of Clinical Psychology, 58(12), 1465-1487. doi:10.1002/jclp.10099

Kosminsky, P.S., \& Jordan, J. J. (2016). Attachmentinformed grief therapy: The clinician's guide to foundations and applications. Routledge.
Luber, M., \& Shapiro, F. (2009). Protocol for excessive grief. In M. Luber (Ed.), Eye movement desensitization and reprocessing (EMDR) scripted protocols: Basics and special situations (pp. 175-187). Springer Publishing.

Marwit, S. J., \& Klass, D. (1996). Grief and the role of the inner representation of the deceased. In D. Klass, P. R. Silverman, \& S. Nickman (Eds.), Continuing bonds (pp. 297-309). Taylor and Francis.

Meier, A. M., Carr, D. R., Currier, J., \& Neimeyer, R. A. (2013). Attachment anxiety and avoidance in coping with bereavement: Two studies. Journal of Social and Clinical Psychology, 32(3), 315-334. doi:10.1521/jscp.2013.32.3.315

Meysner, L., Cotter, P., \& Lee, C. (2016). Evaluating the efficacy of EMDR with grieving individuals: A randomized control trial. Journal of EMDR Practice and Research, 10(1), 1-11. doi:10.1891/1933-3196.10.1.2

Mikulincer, M., \& Shaver, P. R. (2016). Attachment in adulthood: Second edition: Structure, dynamics, and change. Guilford Press.

Mol, S. S. L., Arntz, A., Metsemakers, J. F. M., Dinant, G., Vilters-Van Montfort, P. A. P., \& Knottnerus, A. (2005). Symptoms of post-traumatic stress disorder after non-traumatic events: Evidence from an open population study. British Journal of Psychiatry, 186, 494499. doi:10.1192/bjp.186.6.494

Murray, K. (2012). EMDR with grief: Reflections on Ginny Sprang's 2001 study. Journal of EMDR Practice and Research, 6(4), 187-191. doi:10.1891/1933-3196.6.4.187

Neimeyer, R. A. (2015). Treating complicated bereavement: The development of grief therapy. In J. M. Stillion $\&$ T. Attig (Eds.), Death dying, and bereavement: Contemporary perspectives, institutions and practices (pp. 307-320). Springer Publishing.

Osterweis, M., Solomon, F., \& Green, M (Eds.). (1984). Bereavement: Reactions, consequences, and care. Washington, DC: National Academy Press.

Parkes, C. M. (2013). Love and loss: The roots of grief and its complications. Routledge/Taylor and Francis Group.

Parkes, C. M., \& Prigerson, H. G. (2010). Bereavement: Studies of grief in adult life (4th ed.). Routledge, Taylor $\&$ Francis Group.

Pearlman, L. A., Wortman, C. B., Feuer, C. A., Farber, F. H., \& Rando, T. A. (2014). Treating traumatic bereavement. Guilford Press.

Prigerson, H. G., Vanderwerker, L. C., \& Maciejewski, P. K. (2008). A case for the inclusion of prolonged grief disorder in DSM-V. In M. Stroebe, R. Hansson, H. Schut, \& W. Stroebe (Eds.), Handbook of bereavement research and practice: 21st century perspectives (pp. 165-186). APA.

Rando, T. A. (1993). Treatment of complicated mourning. Research Press.

Rando, T.A., (2015) When trauma and loss collide: The evolution of intervention for traumatic bereavement. In T. Attig \& J. Stillion (Eds.), Death, dying and bereavement: Contemporary perspectives, institutions 
and practices (pp. 321-334). Springer Publishing Company.

Shapiro, F. (1995). Eye movement desensitization and reprocessing: Basic principles, protocols and procedures. Guilford Press.

Shapiro, F. (2001). Eye movement desensitization and reprocessing: Basic principles, protocols and procedures (2nd ed.). Guilford Press.

Shapiro, F. (2018). Eye movement desensitization and reprocessing: Basic principles, protocols and procedures (3rd ed.). Guilford Press.

Shear, K., \& Shair, H. (2005). Attachment, loss, and complicated grief. Developmental Psychobiology, 47, 253-267. doi: $10.1002 /$ dev.20091

Solomon, R. M. (in press). Utilization of EMDR therapy with grief and mourning. Raffaello Cortina.

Solomon, R. M., \& Rando. T. (2007). Utilization of EMDR in the treatment of grief and mourning. Journal of EMDR Practice and Research, 1, 109-117. doi:10.1891/1933-3196.1.2.109

Solomon, R., \& Rando, T. (2012). Treatment of grief and mourning through EMDR: Conceptual considerations and clinical guidelines. European Review of Applied Psychology, X, 231-239. doi:10.1016/j.erap.2012.09.002

Solomon, R. M., \& Rando, T. A. (2015). EMDR therapy and grief and mourning. In M. Luber (Ed.), Eye movement desensitization and reprocessing (EMDR) therapy scripted protocols and summary sheets: Treating trauma and stressor related conditions (pp. 230-252). Springer Publishing.

Solomon, R.M., (2018). EMDR treatment of grief and mourning. Clinical Neuropsychiatry (2018) 15, 3, 173-186.

Solomon, R. M., \& Shapiro, F. (1997). Eye movement desensitization and reprocessing: An effective therapeutic tool for trauma and grief. In C. Figley, B. Bride, \& M. Nicholas (Eds.), Death and trauma (pp. 231-247). Taylor and Francis.

Sprang, G. (2001). The use of eye movement desensitization and reprocessing (EMDR) in the treatment of traumatic stress and complicated mourning: Psychological and behavioral outcomes. Research on Social Work Practice, 11, 300-320. doi:10.1177/ 104973150101100302

Stroebe, M. S., \& Schut H. (1999). The dual process model of coping with bereavement: Rationale and description. Death Studies 23, 3, 197-224.
Stroebe, M. S., \& Schut, H. (2010). The dual process model of coping with bereavement: a decade on. Omega: Journal of Death and Dying 61, 4, 273-289.

Stroebe, M., \& Schut, H. (2008). The Dual Process Model of coping with bereavement: Overview and update. Grief matters. The Australian Journal of Grief and Bereavement, $11,1-4$.

Wang, C., Pan, R., Wan, X., Tan, Y., Xu, L., Ho, C. S., \& ... Ho, R. C. (2020). Immediate psychological responses and associated factors during the initial stage of the 2019 coronavirus disease (COVID-19) epidemic among the general population in China. International Journal Environmental Research Public Health, 17(5), 40-48. doi:10.3390/ijerph17051729

Wayment, H. A., \& Vierthaler, J. (2002). Attachment style and bereavement reactions. Journal of Loss and Trauma, 7(2), 129-149. doi:10.1080/153250202753472291

Worden, J. W. (2018). Grief counseling and grief therapy: A handbook for the mental health practitioner. Springer Publishing.

Yasar, A. B., Abamor, A. E., Usta, F. D., Tay-can, S. E., \& Eroglu, M. Z. (2017). A case study: Effects of EMDR therapy on a patient with persistent complex bereavement disorder (PCBD). European Psychiatry, 41, S728. doi:10.1016/j.eurpsy.2017.01.1326

Disclosure. The authors have no relevant financial interest or affiliations with any commercial interests related to the subjects discussed within this article.

Funding. The authors received no specific grant or financial support for the research, authorship, and/or publication of this article.

Correspondence regarding this article should be directed to Roger M. Solomon, 4001 9th Street North, \#404 Arlington, VA 22202. E-mail: rogermsolomon@aol.com 